\title{
Plasma-type gelsolin in subarachnoid hemorrhage: novel biomarker today, therapeutic target tomorrow?
}

\author{
Sherry Hsiang-Yi Chou ${ }^{1,2}$, Eng H Lo ${ }^{2}$ and MingMing Ning ${ }^{2 *}$ \\ See related research by Pan et al., http://ccforum.com/content/17/4/R149
}

\begin{abstract}
There is growing interest in the potential neuroprotective properties of gelsolin. In particular, plasma-type gelsolin (pGSN) can ameliorate deleterious inflammatory response by scavenging pro-inflammatory signals such as actin and lipopolysaccharide. In a recent issue of Critical Care, Pan and colleagues report an important association between $\mathrm{PGSN}$ and subarachnoid hemorrhage (SAH) disease severity, and found PGSN to be a novel and promising biomarker for SAH clinical outcome. Previous research shows pGSN may be actively degraded by neurovascular proteases such as matrix metalloproteinases in the cerebral spinal fluid of SAH patients. Taken together, these results suggest that pGSN is not only a novel marker of SAH clinical outcome, but may also play an active mechanistic role in $\mathrm{SAH}$, and potentially serve as a future therapeutic target.
\end{abstract}

In a recent issue of Critical Care, Pan and colleagues [1] report the largest study to date on blood plasma-type gelsolin (pGSN) as a promising biomarker in subarachnoid hemorrhage (SAH). In a cohort of 262 well characterized SAH patients and 150 control subjects, Pan and colleagues found that low blood pGSN (compared to healthy controls) is independently predictive of poor outcome or death [1]. A pGSN level $<63.3 \mathrm{mg} / \mathrm{L}$ has $80.8 \%$ sensitivity and $75.1 \%$ specificity in predicting poor neurologic outcome at 6 months. They also found an important association between the level of gelsolin and severity of SAH, as measured by World Federation of Neurosurgical Societies and Fisher grading scales [1].

\footnotetext{
* Correspondence: ning@hms.harvard.edu

2Department of Neurology, Massachusetts General Hospital, Harvard Medical School, 15 Parkman Street, Boston, MA 02114, USA

Full list of author information is available at the end of the article
}

There is growing interest in the potential neuroprotective properties of gelsolin, which has two distinct isoforms. Cytoplasmic gelsolin (cGSN) is intracellular and ubiquitously expressed by all tissues, while pGSN has a 23 amino acid moiety targeted for extracellular secretion [2,3]. Though cGSN has been implicated in numerous biological processes, including cell motility, apoptosis, and phagocytosis [4], the function of pGSN remains largely unknown. Since pGSN can scavenge circulating actin and lipopolysaccharide, it is thought that pGSN may dampen the deleterious pro-inflammatory effects of these circulating inflammatory mediators [4].

Animal data have suggested a neuroprotective role for gelsolin since gelsolin-null neurons enhance cell death and gelsolin-null mice have larger infarct sizes following ischemia [5]. Gelsolin-null cells and animals are deficient in both cGSN and pGSN. Subsequent experiments showed that local infusion of pGSN decreases infarct size after ischemia [6], suggesting pGSN has a pivotal role in stroke.

Human data have consistently shown that pGSN is decreased in severe illnesses, including sepsis [7], and that lower blood pGSN is associated with higher mortality [8]. In neurovascular disorders, pGSN is decreased in both blood and cerebral spinal fluid (CSF) in SAH [9], and decreased blood pGSN is independently associated with mortality after intracerebral hemorrhage and ischemic stroke $[10,11]$. However, the fact that pGSN is associated with a wide spectrum of illnesses raises the concern that blood pGSN depletion is a non-specific marker of disease severity.

Is there any evidence that pGSN may be mechanistically involved in SAH? Indeed, SAH is the only condition where pGSN has been identified in the CSF compartment itself [9]. In SAH, novel degraded pGSN fragments were also identified in the CSF - likely resulting from 
active digestion by matrix metalloproteinases (MMPs) [9], the elevation of which is associated with worse $\mathrm{SAH}$ outcome [12].

In their study, Pan and colleagues very nicely demonstrated a dose-dependent effect of pGSN on SAH disease severity - the lower the level of pGSN, the more severe the SAH grade and the worse the clinical outcome [1]. Previous research has shown pGSN to be present in the CSF compartment in SAH and that MMPs may mediate the depletion of pGSN $[9,12]$. Taken together, these studies suggest that, in the case of SAH, pGSN may be more than just a non-specific marker. It may play an active mechanistic role in SAH, and could potentially serve as a therapeutic target.

Many questions still remain in the gelsolin story in SAH. Exactly how does blood pGSN affect SAH outcome? Where is the source of pGSN in the CSF - does it come from blood? How does the pGSN-MMP pathway facilitate inflammation and injury in the central nervous system and blood? Further targeted mechanistic studies and collaborative multicenter translational and clinical studies are needed to answer these important questions. Indeed, pGSN may not only be a novel and important clinical biomarker for SAH clinical outcome, but may also be a future potential target for treatment.

\section{Abbreviations}

cGSN: Cytoplamic gelsolin; CSF: Cerebral spinal fluid; MMP: Matrix metalloproteinase; pGSN: Plasma-type gelsolin; SAH: Subarachnoid hemorrhage.

\section{Competing interests}

The authors declare that they have no competing interests.

\section{Author details}

'Brigham and Women's Hospital, Department of Neurology, 45 Francis St, Suite BB-204, Boston, MA 02115, USA. ²Department of Neurology, Massachusetts General Hospital, Harvard Medical School, 15 Parkman Street, Boston, MA 02114, USA.

Published: 06 Jan 2014

\section{References}

1. Pan JW, He LN, Xiao F, Shen J, Zhan RY: Plasma gelsolin levels and outcomes after aneurysmal subarachnoid hemorrhage. Crit Care 2013, 17:R149.

2. Kwiatkowski DJ, Stossel TP, Orkin SH, Mole JE, Colten HR, Yin HL: Plasma and cytoplasmic gelsolins are encoded by a single gene and contain a duplicated actin-binding domain. Nature 1986, 323:455-458.

3. Yin HL, Kwiatkowski DJ, Mole JE, Cole FS: Structure and biosynthesis of cytoplasmic and secreted variants of gelsolin. J Biol Chem 1984, 259:5271-5276.

4. Li GH, Arora PD, Chen Y, McCulloch CA, Liu P: Multifunctional roles of gelsolin in health and diseases. Med Res Rev 2010 [Epub ahead of print].

5. Endres M, Fink K, Zhu J, Stagliano NE, Bondada V, Geddes JW, Azuma T, Mattson MP, Kwiatkowski DJ, Moskowitz MA: Neuroprotective effects of gelsolin during murine stroke. J Clin Invest 1999, 103:347-354.

6. Le HT, Hirko AC, Thinschmidt JS, Grant M, Li Z, Peris J, King MA, Hughes JA, Song S: The protective effects of plasma gelsolin on stroke outcome in rats. Exp Trans/ Stroke Med 2011, 3:13.
7. Lee PS, Patel SR, Christiani DC, Bajwa E, Stossel TP, Waxman AB: Plasma gelsolin depletion and circulating actin in sepsis: a pilot study. PLOS One 2008, 3:e3712.

8. Lee PS, Drager LR, Stossel TP, Moore FD, Rogers SO: Relationship of plasma gelsolin levels to outcomes in critically ill surgical patients. Ann Surg 2006, 243:399-403.

9. Chou SH, Lee PS, Konigsberg RG, Gallacci D, Chiou T, Arai K, Simmons S, Bauer D, Feske SK, Lo EH, Ning MM: Plasma-type gelsolin is decreased in human blood and cerebrospinal fluid after subarachnoid hemorrhage. Stroke 2011, 42:3624-3627.

10. Guo XC, Luo BY, Li XF, Yang DG, Zheng XN, Zhang K: Plasma gelsolin levels and 1-year mortality after first-ever ischemic stroke. J Crit Care 2011, 26:608-612.

11. Zhao DQ, Wang K, Zhang HD, Li YJ: Significant reduction of plasma gelsolin levels in patients with intracerebral hemorrhage. Clin Chim Acto 2013, 415:202-206.

12. Chou SH, Feske SK, Simmons SL, Konigsberg RG, Orzell SC, Marckmann A, Bourget G, De Jager PL, Du R, Arai K, Lo EH, Ning MM: Elevated peripheral neutrophils and matrix metalloproteinase 9 as biomarkers of functional outcome following subarachnoid hemorrhage. Trans/ Stroke Res 2011, 2:600-607.

\section{$10.1186 / \mathrm{cc} 13178$}

Cite this article as: Chou et al:: Plasma-type gelsolin in subarachnoid hemorrhage: novel biomarker today, therapeutic target tomorrow?. Critical Care 2014, 18:101 